\title{
Electric field-triggered Cassie-Baxter-Wenzel wetting transition on textured surface
}

\author{
Ke Xiao, ${ }^{*}$ Xi Chen, ${ }^{*}$ and Chen-Xu Wu $\odot^{\dagger}$ \\ Fujian Provincial Key Laboratory for Soft Functional Materials Research, Department of Physics, College of Physical Science \\ and Technology, Xiamen University, Xiamen 361005, People's Republic of China
}

(Received 27 January 2021; accepted 12 August 2021; published 24 September 2021)

\begin{abstract}
Understanding the critical condition and mechanism of the droplet wetting transition between Cassie-Baxter state and Wenzel state triggered by an external electric field is of considerable importance because of its numerous applications in industry and engineering. However, such a wetting transition on a patterned surface is still not fully understood, e.g., the effects of electrowetting (EW) number, geometry of the patterned surfaces and droplet volume on the transition have not been systematically investigated. In this paper, we propose a theoretical model for the Cassie-Baxter-Wenzel wetting transition triggered by an external voltage applied to a droplet placed on a mirco-pillared surface or a porous substrate. It is found that the external field applied lowers the energy barrier for the droplet to cross over and complete the wetting transition. Our calculations also indicate that for a fixed droplet volume, the critical EW number (voltage) will increase (decrease) along with the surface roughness for a micropillar-patterned (porous) surface, and if the surface roughness is fixed, a small droplet tends to ease the critical EW condition for the transition. Besides, three-dimensional phase diagrams in terms of EW number, surface roughness, and droplet volume are constructed to illustrate the wetting transition. Our theoretical model can be used to explain the previous experimental results about the Cassie-Baxter-Wenzel wetting transition reported in the literature.
\end{abstract}

DOI: 10.1103/PhysRevResearch.3.033277

\section{INTRODUCTION}

Wettability is widely studied in order to gain highly waterrepellent substrates referred to as superhydrophobic surfaces with an ultrahigh apparent contact angle, a much smaller contact angle hysteresis [1,2], and hydrodynamic slip [3-5]. These properties rely on the nano- or the microscale topological structures of such surfaces, which exhibit a broad range of applications in engineering such as self-cleaning [6,7], water proofing [8,9], drag reduction [10], anti-dew/reflection $[11,12]$, bactericidal activity [13-16], and so on. Typically, when a liquid droplet rests on such a roughened surface, a classical description of the droplet is characterized by Wenzel (W) [17] and Cassie-Baxter (CB) [18] wetting states. The former corresponds to a homogenous wetting state in which liquid percolates into the texture, i.e., a fully wetted state losing superhydrophobicity, whereas the droplet in the CB state merely suspends on the tips of the rough surface, featured with air being trapped in the cavities of the rough surface topography. Generally, the transition between these two states can be triggered via various approaches by tuning external control parameters, such as passive strategies that rely, for ex-

\footnotetext{
*These authors contributed equally to this work.

†cxwu@xmu.edu.cn

Published by the American Physical Society under the terms of the Creative Commons Attribution 4.0 International license. Further distribution of this work must maintain attribution to the author(s) and the published article's title, journal citation, and DOI.
}

ample, on the utilization of gravity force [19,20], evaporation [21-23], and Laplace pressure [24,25], and active strategies that employ surface acoustic wave [26], vibration [27,28], and even electric field [29-32], a technique called electrowetting $(\mathrm{EW})$. Its adaption to various geometries, little power consumption, and fast and precise fine-tuning of the wetting state makes it a prevalent technique receiving significant research interest in the past decade [33,34]. It is well known that once an electric voltage is applied between a substrate and a droplet settling on it, the initial equilibrium contact angle of the droplet will be reduced to a new smaller value, leading to an alteration of its apparent wettability referred to as EW-on-dielectric $[33,35]$. Such an EW phenomenon has attracted significant attention due to its extensive applications in laboratory-on-a-chip systems [33] and microfluidic operations $[36,37]$ and the ability to induce wetting state transition [29-32] and droplet detachment [38-41].

Recently, numerous efforts of experiments [29,42,43], theoretical modelings [31,37], and computer simulations $[30,32,44]$ have been devoted to getting a better understanding of the wetting transition triggered by electric field. By combining experimental results and numerical simulations, it has been found that the stability of the CB state under EW is determined by the balance of the Maxwell stress and the Laplace stress [29]. Meanwhile, the wetting transition from $\mathrm{CB}$ state to $\mathrm{W}$ state is controlled by the energy barrier stemming from the pinning of the contact lines at the edges of the hydrophobic pillars [29]. Based on a surface energy model, Roy et al. estimated the energy barriers for the EW-induced CB-to-W transition of a droplet on mushroomshaped re-entrant microstructures and an array of cylindrical 
microposts. They experimentally demonstrated that the transition on a mushroom structure is more resilient than that on an array of microposts [31]. The authors assumed that for a droplet in the composite state, the liquid-vapor interface infiltrating a unit cell is a spherical interface with its radius and Young's contact angle made with the micropost walls remaining unchanged. Besides, computer simulation also provides a useful complement to revealing the underlying mechanism of droplet wetting transition on textured surfaces. By employing molecular dynamics simulations, Zhang et al. [32] studied the mechanism behind the CB-to-W transition for a nanoscale water droplet resting on a nanogrooved surface in the presence of an external electric field and found that there exists an energy barrier separating the CB state and the W state. In addition, they also discussed the dependence of the energy barrier on the electric field strength, the groove aspect ratio, and the intrinsic contact angle of the groove.

Despite the fact that the EW-induced transition has been extensively studied via either experimental or theoretical approaches, a systematic analytical understanding of the underlying mechanism, in particular, the dependence of critical electric voltage on surface roughness and droplet volume, has not been explored.

In this paper, we establish a theoretical model to study the EW transition on micropatterned surfaces through analyzing the difference in interfacial free energy between the CB state and the intermediate composite state. The effects of surface roughness and droplet volume on the threshold voltage are discussed. To further explore the interrelation among threshold voltage, surface roughness and droplet volume, three-dimensional (3D) phase diagrams in the corresponding parameter space are also constructed. We expect that our model can offer some guidance to the design and fabrication of patterned surfaces and allow one to study EW transition on other types of patterned surfaces.

\section{THEORETICAL MODELING}

We begin our investigation by considering an EW setup consisting of a millimeter-sized sessile water droplet deposited on two different types of superhydrophobic surfaces decorated with, respectively, a square lattice of cylindric mircropillars and a square array of pores, as shown in Fig. 1. The bottom surface consists of an insulating surface layer (thickness $d$ ) on top and an electrode underneath. The 3D geometry of the micropillar-patterned surface in this paper is schematically shown in Fig. 1(a). The top view and the side view of the squarely distributed micropore-patterned surface are sketched in Figs. 1(b) and 1(c), respectively. Here, the cylindrical pillars and the pores are characterized by their radius $R$, height $H$, and gap pitch $P$ (or center-to-center interspacing $S$ ) between neighboring pillars or pores. Traditionally, nondimensional parameters, roughness factor $r$, and solid fraction $\phi_{s}$, which are defined as the ratio of the actual area of the solid surface to its projection area and the ratio of the contact solid surface (tip of the pillars or the pores) to the total horizontal surface, respectively, are commonly used to represent the level of roughness for the textured surfaces. Geometrically, in this paper, the roughness factor is given by $r=1+2 \pi R H / S^{2}$, and the solid fractions $\phi_{s}$ are written as
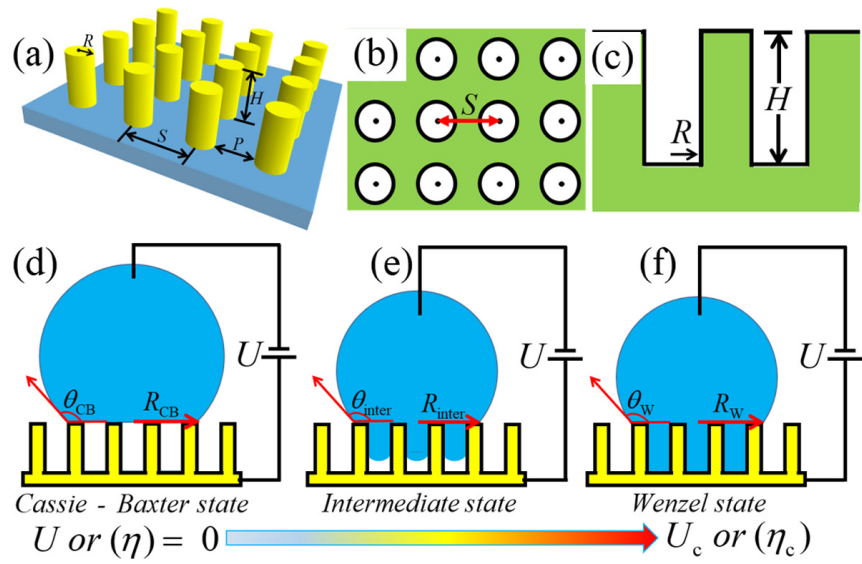

FIG. 1. (a) Schematic 3D picture of a microstructured surface with cylindrical pillars of radius $R$, height $H$, and gap pitch $P$ on a periodic square lattice with pillar-to-pillar spacing $S$. Schematic (b) top view and (c) side view of a regular array of pores of radius $R$, depth $H$, gap pitch $P$, and center-to-center pore spacing $S$. Different wetting states of a droplet on a microtextured surface in the presence of an external electric voltage: (d) CB state, (e) intermediate composite state, and (f) W state.

$\pi R^{2} / S^{2}$ for the pillar-patterned surface and $1-\pi R^{2} / S^{2}$ for the porous surface, respectively. The two possible wetting states, i.e., the $\mathrm{CB}$ state and the $\mathrm{W}$ state, are illustrated by Figs. 1(d) and 1(f). Between them there exists an energy barrier, i.e., an unstable intermediate composite state [see Fig. 1(e)], which can be lowered to a level below the CB state by applying an external electric voltage across the droplet. This leads to a CB-to-W wetting transition. In order to probe the critical electric voltage that triggers the wetting transition, it is necessary to calculate, for the CB state and the intermediate composite state, the Gibbs free energy [33]

$$
G=\gamma_{\mathrm{lv}} A_{\mathrm{lv}}+\gamma_{\mathrm{sv}} A_{\mathrm{sv}}+\gamma_{\mathrm{ls}}^{\text {eff }} A_{\mathrm{ls}}-\Delta P_{0} V_{\mathrm{l}},
$$

which consists of interfacial free energies and a bulk energy contributed by pressure. Here $A_{\mathrm{lv}}, A_{\mathrm{sv}}$, and $A_{\mathrm{ls}}$ are the areas of the liquid-vapor, solid-vapor, and liquid-solid interfaces, and $\gamma_{\mathrm{lv}}, \gamma_{\mathrm{sv}}$, and $\gamma_{\mathrm{ls}}^{\text {eff }}$ are the liquid-vapor, solid-vapor, and effective liquid-solid interfacial energy densities, respectively; $\gamma_{\mathrm{ls}}^{\text {eff }}=\gamma_{\mathrm{ls}}-\eta \gamma_{\mathrm{lv}}$ with $\eta=\varepsilon_{0} \varepsilon U^{2} / 2 d \gamma_{\mathrm{lv}}$ represents the dimensionless EW number, where $\varepsilon_{0}, \varepsilon$, and $d$ are the dielectric permittivity in vacuum, the relative dielectric constant, and the thickness of the insulating layer, respectively. $V_{1}$ is the volume of the droplet, and $\Delta P_{0}$ is the pressure difference across the liquid-vapor interface. It is a constant which does not change the free-energy profile $[33,45,46]$. In addition to $\Delta P_{0}$, theoretically the Maxwell stress $\epsilon \epsilon_{0} E^{2} / 2$ close to the triple contact line also makes a contribution to the force balance across the liquid-vapor interface, which introduces an equivalent Laplace pressure $\Delta P$ given by [33]

$$
\Delta P=\Delta P_{0}+\frac{1}{2} \epsilon \epsilon_{0} E^{2} .
$$

A comparison between Maxwell stress and $\Delta P_{0}$ shows that the contribution of the external field to sagging is trivial (three orders smaller than $\Delta P_{0}$ ), and thus we may assume that sagging is mainly driven by pressure difference across the interface, while the external field plays a critical role in the 
depinning process. As in this paper, the liquid-vapor interface, which hangs over the micropillar side walls, is approximated as a spherical cap with an equivalent base radius and makes a fixed Young's contact angle with the micropillar walls during the depinning process $[31,47,48]$. A more detailed explanation can be found in the Appendix. It should be noted that as both the pressure difference $\Delta P_{0}$ and the droplet volume $V_{1}$ are independent of the external field, we may simply ignore the contribution of $\Delta P_{0} V_{1}$ in our calculation. In this sense, we focus our investigation of the CB-W transition on the depinning process. Let $A_{\mathrm{t}}=A_{\mathrm{sv}}+A_{\mathrm{ls}}$, and with a consideration of the Young's equation $\gamma_{\mathrm{sv}}-\gamma_{\mathrm{ls}}=\gamma_{\mathrm{lv}} \cos \theta_{\mathrm{Y}}$, Eq. (1) can be converted to

$$
G=\gamma_{\mathrm{lv}} A_{\mathrm{lv}}-\gamma_{\mathrm{lv}}\left(\cos \theta_{\mathrm{Y}}+\eta\right) A_{\mathrm{ls}}+\gamma_{\mathrm{sv}} A_{\mathrm{t}},
$$

where $\theta_{\mathrm{Y}}$ is the apparent contact angle at the equilibrium state.

In the present study, we assume that the volume of the droplet is small, that is, with a characteristic size smaller than the capillary length $l_{\mathrm{c}}=\sqrt{\gamma_{\mathrm{lv}} / \rho g} \sim 2.7 \mathrm{~mm}$, where $\rho$ and $g$ are the density of the water and the gravitational acceleration, respectively. In this case the gravitational effect can be neglected, and the shape of the droplet associated with the transition can be treated as a sphere. Therefore, the total interfacial free energy of a water droplet in CB state on a patterned surface, as schematically illustrated by Fig. 1(d), can be calculated as

$$
\begin{aligned}
G_{\mathrm{CB}}= & \gamma_{\mathrm{lv}} \pi R_{\mathrm{CB}}^{2}\left[v\left(\theta_{\mathrm{CB}}\right)+\left(1-\phi_{s}\right)-\left(\cos \theta_{\mathrm{Y}}+\eta\right) \phi_{s}\right] \\
& +\gamma_{\mathrm{sv}} A_{\mathrm{t}},
\end{aligned}
$$

where $v(\theta)=2 /(1+\cos \theta)$ is a dimensionless function. In addition, as the evaporation effect of the water droplet is excluded as well, it is reasonable to assume that the droplet volume $V_{0}$ is conserved. Therefore, the base radius $R_{\mathrm{CB}}$ and the contact angle $\theta_{\mathrm{CB}}$ of the droplet, which depends on the external field applied, can be determined by minimizing the global energy under the constraint of fixed droplet volume. Similarly, the total interfacial free energy of the intermediate composite state reads

$$
\begin{aligned}
G_{\text {inter }}= & \gamma_{\text {lv }} \pi R_{\text {inter }}^{2}\left\{v\left(\theta_{\text {inter }}\right)+\left(1-\phi_{s}\right) v\left(\theta_{\mathrm{Y}}-\frac{\pi}{2}\right)\right. \\
& \left.-\left(\cos \theta_{\mathrm{Y}}+\eta\right)\left[\phi_{s}+(r-1) \frac{h}{H}\right]\right\}+\gamma_{\mathrm{sv}} A_{\mathrm{t}},
\end{aligned}
$$

where $R_{\text {inter }}$ and $h$ are the base radius of the droplet and the penetration height from the tip of the pillars or pores to the point at which the curved interstitial liquid-vapor meniscus touches the edges of the walls (see Appendix for the calculation of $h$ ), respectively. For a droplet at the $\mathrm{W}$ state, the total interfacial free energy becomes

$$
G_{\mathrm{W}}=\gamma_{\mathrm{lv}} \pi R_{\mathrm{W}}^{2}\left[v\left(\theta_{\mathrm{W}}\right)-\left(\cos \theta_{\mathrm{Y}}+\eta\right) r\right]+\gamma_{\mathrm{sv}} A_{t},
$$

where $\theta_{\mathrm{W}}$ and $R_{\mathrm{W}}$ are, respectively, the contact angle and the base radius of the spherical cap above the structured surface determined by a minimization of the total energy.

In order to study the influence of an EW voltage on the state of a droplet on a grooved surface, Bahadur and Garimella [37] employed an energy-minimization-based modeling approach to analyze the dependence of the apparent contact angle on an
EW voltage and to investigate the $\mathrm{CB}-\mathrm{W}$ transition under the influence of an EW voltage and estimate the energy barrier. In this paper, using a similar approach to that of Refs. [31] and [37], on a pillared surface and a pored surface, respectively, we comprehensively consider both the sagging and the variation of the contact angle and the base radius, which is determined by a global energy minimization. In addition, we explore the dependence of the critical transition voltage not only on the surface roughness but also on the droplet size.

Determining the wetting path is of particular importance to understand wetting transition. In this sense, how to obtain the free-energy landscape as a function of the wetting process becomes critical. Ren [49] studied the wetting transition on microstructured hydrophobic surfaces via accurately computing the transition states, the energy barriers, and the minimum energy paths with the climbing string method. They focused on the overdamped dynamics perturbed by a small noise to track the saddle points of the energy, which in the transition state theory represents the dynamical bottlenecks for the transition states. The results showed that the wetting was initiated by an infiltration of liquid in a single cavity, followed by stepwise propagation of the liquid front on the solid surface. The dependence of the energy barriers on the pillar height and the drop size was also discussed. By carrying out rare-event atomistic simulations, Amabili et al. [50] investigated the process of CB-W state transition on a nanopillared surface. It was found that the collapse of the superhydrophobic $\mathrm{CB}$ state started from the formation of a liquid finger between two pillars, which was found to be the transition state determining the free-energy barrier, following a progressively wetting of the surrounding interpillar spaces, and the mechanism could not be captured by previous simulations. There are also other numerical and simulation methods to study the wetting transition, such as MD simulations [51], the lattice Boltzmann methods [52], the minimum energy paths computation method [53], and the computational fluid dynamics (CFD) approach [54]. The literature all focused on investigating the wetting transition in the absence of an external electric field. However, how the effects of surface roughness and droplet size on the critical electric voltage of wetting transition induced by EW still remain unclear.

So far, there have been a couple of reports on the mechanisms triggering $\mathrm{CB}-\mathrm{W}$ wetting transition on structured surfaces. In the absence of an external field, Patankar proposed two possible mechanisms, depinning and sagging, for the CB-to-W transition due to Laplace pressure [48]. The predicted contact angle at the liquid-air interface due to depinning based on their model is found to be greater than the maximum contact angle that can be sustained at a corner. On the other hand, the wetting transition from $\mathrm{CB}$ state to $\mathrm{W}$ state also occurs when the sag of the curved liquid-air interface touches the bottom of the rough-pillared surface. Such a sag mechanism is significantly influenced by pillar diameter, spacing between pillars, and droplet volume [55]. Recently, by combining the thermodynamic approach and a CFD approach, Rohrs et al. [54] investigated the wetting phenomena on ideal micropatterned surfaces consisting of straight micropillars at different pillar dimensions and spacing. In their theoretical model, they assumed that there exists a maximum sagging between the pillar valleys when the droplet is in a partial 

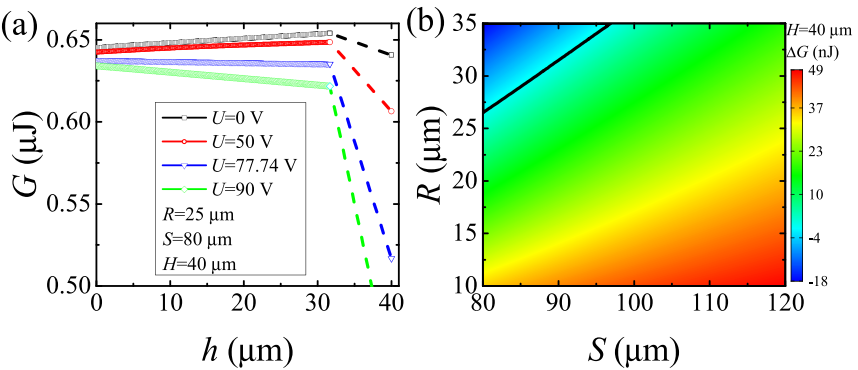

FIG. 2. (a) Variation of the total interfacial free energy with respect to the invading depth. (b) Theoretical wetting phase diagram in the parameter space spanned by $R$ and $S$ for a droplet placed on a micropillar-patterned surface.

penetrating state. In this paper, we approximate the sag as a spherical cap during the depinning process.

Here it should be noted that besides the energyminimization approach, the $\mathrm{CB}-\mathrm{W}$ transition in the absence of an external field can be investigated alternatively via force balance analysis. For submersed superhydrophobic surfaces, Xue et al. [56] investigated the wetting states on submersed hydrophobic surfaces under hydrostatic pressure and found that the stability of different wetting states depends on the hydrostatic pressure. Also, in Ref [57], it was stated that the underlying mechanism of wetting transition is the collapse of liquid-air interfaces when the equilibrium among liquid, air, and capillary pressure is broken. However, if an external field is introduced, such a force balance approach would require a complicated calculation of local electric field distribution.

\section{RESULTS AND DISCUSSION}

In our model, we take into account all the interfacial free energies for a CB state and an intermediate composite state of a 3D droplet placed on a micropillar- or pore-patterned surface. A set of parameters $\gamma_{\mathrm{lv}}=72.8 \mathrm{mN} \cdot \mathrm{m}^{-1}, \varepsilon=3.2$, $d=3 \mu \mathrm{m}$, and $\theta_{\mathrm{Y}}=115^{\circ}$ was chosen for our calculations [31]. The value of dielectric constant corresponds to a layer of the equally transparent SU-8 photoresist used in Ref. [29]. The dependence of total interfacial free energy on the invading depth is presented in Fig. 2, which shows a monostable structure for the CB state. Here, kinks represent the points when the spherical cap touches the bottom of the rough-pillared or pored surface. It is found that there exists an energy barrier separating the $\mathrm{CB}$ state and the $\mathrm{W}$ state [see the square dotted line in Fig. 2(a)] in the presence of a small external field. Once the external electric field exceeds a critical value [see the inverted triangle dotted line in Fig. 2(a)], the energy barrier disappears, triggering a CB-to-W wetting transition. By comparing the total interfacial free energies between the $\mathrm{CB}$ state and the $\mathrm{W}$ state, we constructed an $R-S$ phase diagram consisting of two regions divided by a coexistence line, as shown in Fig. 2(b). In a recent report [58], Li et al. proposed an experimental method by squeezing and releasing a drop between a pillar-patterned surface and a nonadhesive plate to investigate the state transitions between the CB state and the $\mathrm{W}$ state. A phase diagram of the textured material was depicted to distinguish three different regimes as monostability of the CB state, bistability with the metastable CB state,

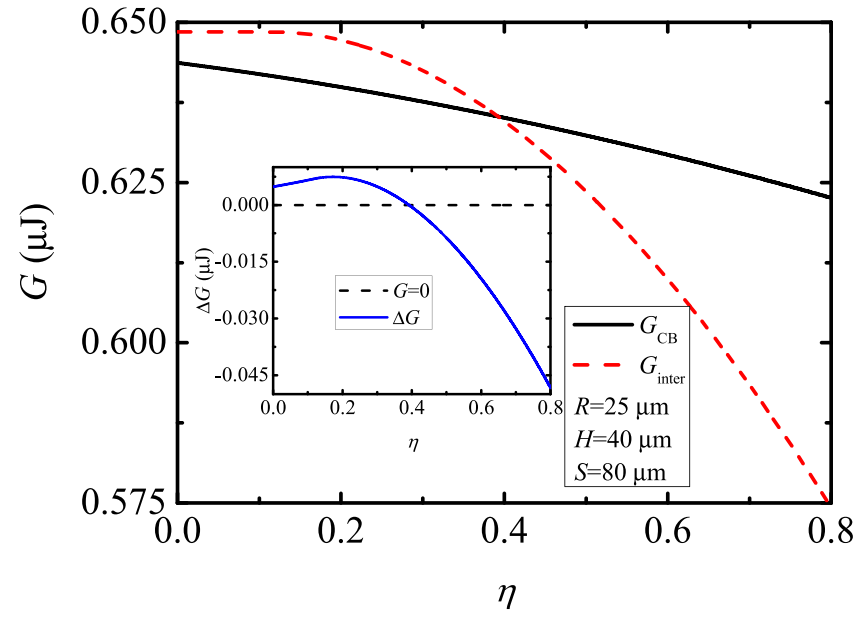

FIG. 3. Representative total interfacial free energies of the CB and the intermediate states of a droplet on a micropillar-patterned surface: $G_{\mathrm{CB}}$ and $G_{\text {inter }}$, respectively, as a function of EW number. The inset shows their difference, $\Delta G=G_{\text {inter }}-G_{\mathrm{CB}}$, which gives the critical EW number (critical external electric voltage) for the wetting transition when $\Delta G=0$.

and bistability with the metastable $\mathrm{W}$ state. The criterions of the two critical lines were formulated as functions of solid fraction, surface roughness, receding angle, and Young's contact angle. Their results show that the monostable CB state can be obtained by considering either a droplet on a doubly textured surface where a nanotexture is added on the pillars or mercury on a simply textured surface.

In order to understand the wetting transition, we compare the energies between the CB state and the intermediate composite state as the external field is applied and increased, as shown in Fig. 3. In the absence of an external field, as valued by the intercept in the figure, the energy of the intermediate composite state is higher than that of the $\mathrm{CB}$ state, denying the occurrence of a CB-to-W transition. Once an external field is applied and increased, it is found that the energy of the intermediate composite state decreases more rapidly than that of the CB state. Therefore there exists an intersection point where the energy barrier disappears, triggering a transition to a W state, as shown in Fig. 3. Such a critical condition corresponds to a critical voltage (or a critical EW number $\eta_{\mathrm{c}}$ ), which can be estimated by equating the energies of these two states for a given textured surface.

For a fixed droplet volume, the EW number $\eta_{\mathrm{c}}$ is found to remarkably depend on the geometric features of the structured surface (surface roughness), such as aspect ratio $R / H$, relative pitch $P / H$ (density $\left.1 / S^{2}=1 /(P+2 R)^{2}\right)$, surface roughness $r$, and solid fraction $\phi_{\mathrm{s}}$, as shown in Fig. 4. As shown by the black square dot curve in Fig. 4(a), it becomes more stringent (easier) for a CB-to-W transition on a micropillar-patterned surface (porous surface) to occur with the increase of its aspect ratio $R / H$. An alternative illustration of Fig. 4(a) is to replace the dimensionless aspect ratio $R / H$ by surface roughness $r$, indicating that roughness suppresses (enhances) EW-induced CB-to-W transition on a micropillar-patterned surface (porous surface) [Fig. 4(b)]. Besides, as the aspect ratio also correlates with solid fraction $\phi_{\mathrm{s}}$, it is also possible to depict the critical 

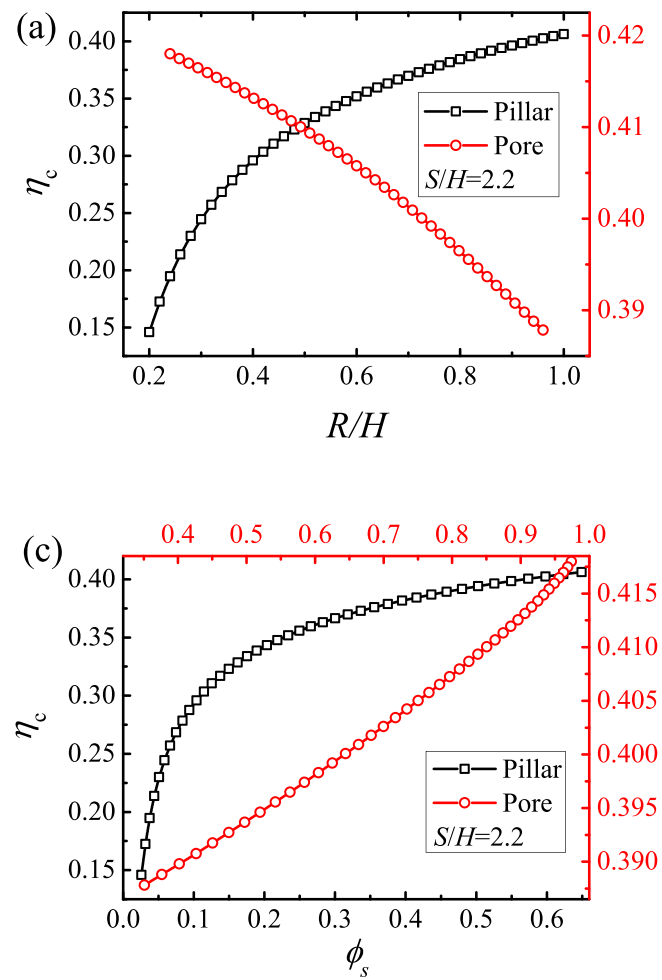
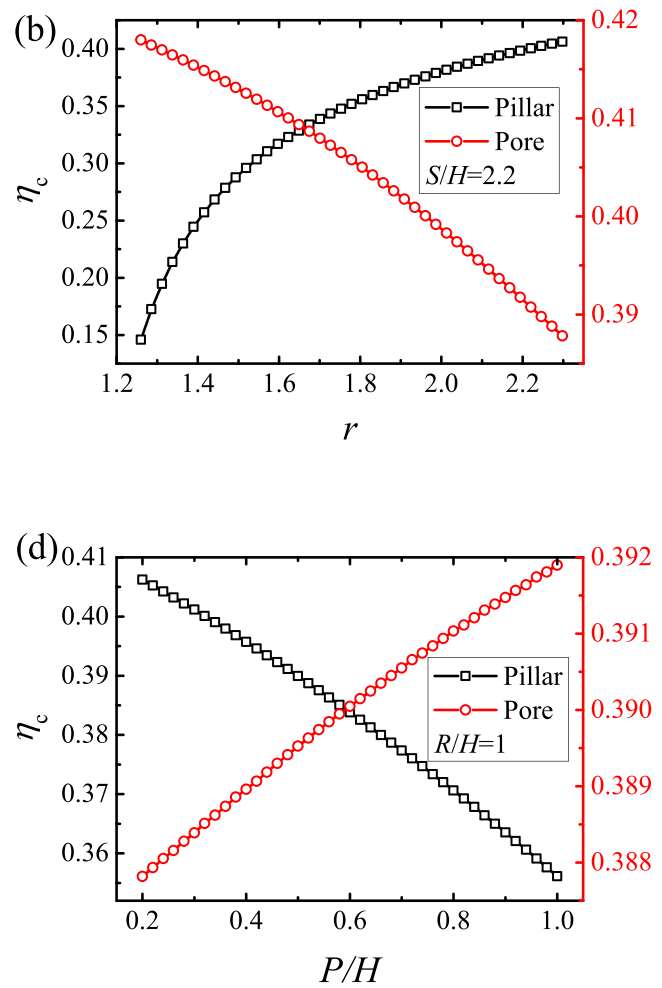

FIG. 4. The critical EW number $\eta_{c}$ for the CB-W transition vs (a) aspect ratio $R / H$, (b) surface roughness $r$, (c) solid fraction $\phi_{s}$, and (d) $P / H$, where the black square dot curve and the red circle dot curve stand for micropillar- and pore-patterned surfaces, respectively.

EW number in terms of solid fraction $\phi_{\mathrm{s}}$ for both pillar- and pore-patterned surfaces, as shown in Fig. 4(c). Apart from the aspect ratio, the distribution densities of pillars and pores also play an important role in determining the critical EW number. A deeper investigation, as shown in Fig. 4(d), exhibits that a reduction of critical EW number $\eta_{\mathrm{c}}$ can be achieved by increasing (decreasing) the pitch of pillar-patterned (porepatterned) surfaces.

According to Eqs. (A1) and (A7), the base radius and the contact angle of the droplet are functions of the droplet

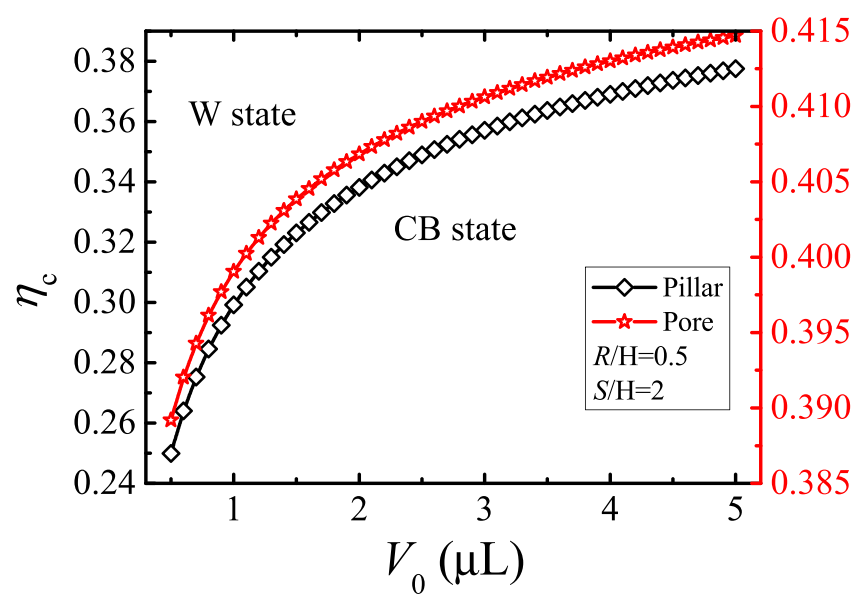

FIG. 5. The critical EW number $\eta_{c}$ for the CB-W transition vs droplet volume $V_{0}$, where the black square dot curve and the red circle dot curve represent micropillar- and pore-patterned surfaces, respectively.

volume under the constraint of fixed droplet volume. As a result, the interfacial energies of both the $\mathrm{CB}$ state [Eq. (4)] and the intermediate state [Eq. (5)] depend on the droplet size. It has been experimentally reported that there exists a critical droplet size for the CB-to-W wetting transition to occur during the evaporation process [21-23], indicating that the favorable wetting state also depends on droplet volume [59]. Such a phenomenon can be explained by our theoretical model. Figure 5 exhibits the effect of droplet volume $V_{0}$ on wetting for a set of fixed surface geometric parameters ( $R=25 \mu \mathrm{m}, H=50 \mu \mathrm{m}$, and $S=100 \mu \mathrm{m})$, revealing that the larger the droplet size, the higher the critical voltage for the wetting transition to occur.

Finally, in order to comprehensively understand how surface roughness and droplet size affect the critical value $\eta_{c}$

(a)

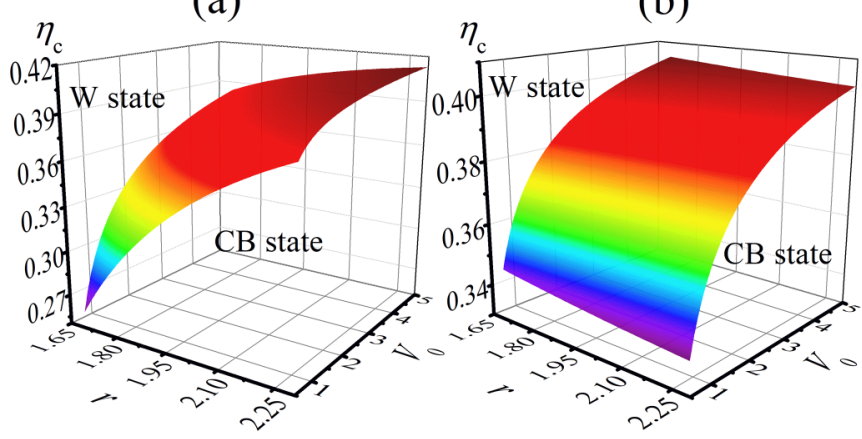

FIG. 6. 3D phase diagrams in terms of the critical EW number $\eta_{c}$, surface roughness $r$, and droplet volume $V_{0}$ for (a) a micropillarpatterned surface and (b) a pore-patterned surface. 
TABLE I. The comparison between the theoretical predictions and the experimental observations.

\begin{tabular}{|c|c|c|c|c|c|c|c|}
\hline \multirow[t]{2}{*}{$V_{0}(\mu \mathrm{L})$} & \multirow[t]{2}{*}{$\varepsilon$} & \multirow[t]{2}{*}{$d(\mu \mathrm{m})$} & \multirow[t]{2}{*}{$r$} & \multirow[t]{2}{*}{$\phi_{s}$} & \multirow[t]{2}{*}{$H(\mu \mathrm{m})$} & \multicolumn{2}{|c|}{ Critical voltage (V) } \\
\hline & & & & & & Experimental & Theoretical \\
\hline \multirow[t]{5}{*}{2.5} & 3.2 & 0.81 & 2.87 & 0.23 & 43.1 & 35 [60] & 41.2 \\
\hline & & & 3.71 & 0.55 & 43.1 & $55[60]$ & 41.9 \\
\hline & & & 2.83 & 0.41 & 24.2 & $58[60]$ & 42.2 \\
\hline & & 1.45 & 2.5 & 0.59 & 23 & $73.3[60]$ & 56.6 \\
\hline & & & 2.75 & 0.21 & 36.5 & $68.3[60]$ & 55.5 \\
\hline \multirow[t]{2}{*}{3} & 3.15 & 3.75 & 1.40 & 0.12 & 30 & $72.4[31]$ & 88.7 \\
\hline & & & 1.42 & 0.13 & 30 & $90.9[31]$ & 88.9 \\
\hline
\end{tabular}

as a whole, 3D phase diagrams for micropillar and porepatterned surface are constructed in terms of EW number, surface roughness, and droplet volume, as demonstrated in Fig. 6. It is found from the $3 \mathrm{D}$ phase diagrams that a higher (lower) critical $\mathrm{EW}$ number $\eta_{c}$ is required to trigger the $\mathrm{CB}-\mathrm{W}$ transition for rougher pillar- (pore-)patterned surfaces and for large droplet as well, regardless of the geometric pattern of the surfaces. Therefore, it becomes possible that the EW-induced CB-to-W transition can be effectively inhibited by engineering a surface with hierarchical roughness or by adopting a large droplet.

To examine the validity of our theoretical model, it is necessary to compare our theoretical predictions with experimental results. For example, Bahadur et al. [60] showed via experiments that the observed transition voltage is $35 \mathrm{~V}$ for a microstructured surface with roughness 2.87 , solid fraction 0.23 , and pillar height $43.1 \mu \mathrm{m}$, while the transition voltage has to be increased to $58 \mathrm{~V}$ to observe the wetting transition for the microstructured surface with same pillar height but an increased surface roughness 3.71 and solid fraction 0.55 , a result qualitatively in good agreement with our present conclusions. In addition, it has been found by experimental observations made by Manukyan et al. [29] that the critical voltage causing the cavities filled with water and the lateral propagation decreases as the gap widths between the pillars were widened (equivalent to a reduction of surface roughness and solid fraction), a conclusion also in accordance with our results. Furthermore, Roy et al. [31] also reported that the more sparsely the cylindrical microposts are distributed, the higher the EW voltage is required to trigger the CB-W transition. By employing molecular dynamics simulations, Zhao et al. [61] investigated the statics and dynamics of EW on pillar-arrayed surfaces at nanoscale, and the presented variation of the critical voltage with respect to the surface roughness and the solid fraction show that the critical voltage increases with the increase of the surface roughness and the solid fraction. This is also in line with our predictions. Recently, Rofman et al. [62] experimentally studied the EW of hierarchical superhydrophobic pillar-patterned surfaces, and they found that the critical EW potential causing the CB-to-W transition decreases linearly with pillar spacing, which shows the same trend as ours in Fig. 4(d). These results all support the theoretical model proposed in this paper qualitatively. Furthermore, in order to make comparisons with experiments quantitatively, we construct the same geometry and configuration of pillars and use the same parameters used in the experimental literature to calculate the critical transition volt- age. The comparison between our theory and the experiments is summarized in Table I. As shown in Table I, the comparison between our results and the experimental observations in Ref. [60] exhibits that the variation trend of the theoretical critical voltage is identical to that of experimental observations, but the critical transition voltage of theoretical predicted is lower than that of experimental values. On the other hand, a comparison between our model results and the experimental observations in Ref. [31] shows that the theoretical predicted critical voltage is higher than the experimental value when $r$ and $\phi_{s}$ are 1.40 and 0.12 . The deviation may be attributed to the contact line friction and the dissipation that are not accounted for in our model. Nevertheless, our model agrees well with the experimental results when $r$ and $\phi_{s}$ take the values of 1.42 and 0.13 , respectively. Our findings may help to understand the $\mathrm{EW}$ on microtextured surfaces and assist the future design of engineered surfaces in practical applications.

\section{CONCLUSION}

In this paper we developed a model to interpret the CB-W wetting transition triggered by an external electric field for a 3D droplet deposited on a micropillar- or pore-patterned surface. It is found that the electric field lowers the energy barrier created by the intermediate composite state, which allows the droplet initially in the CB state to cross the energy barrier and complete the EW-induced CB-W transition. The critical value of the electric field for the transition to occur is influenced by the geometrical parameters of the substrate, such as roughness and solid fraction, and the droplet size. The 3D phase diagrams in terms of EW number, surface roughness, and droplet volume are constructed. It is shown that low (high) roughness, low (high) pitch, small (small) solid fraction, and small (small) droplet size encourage an EW-induced CB-W wetting transition, a conclusion qualitatively in good agreement with previous investigations reported in the literature.

\section{ACKNOWLEDGMENT}

This work was funded by the National Science Foundation of China under Grants No. 11974292 and No. 11947401.

\section{APPENDIX: THE CALCULATION OF THE BASE RADIUS AND THE PENETRATION HEIGHT OF A DROPLET PLACED ON A PATTERNED SURFACE}

For all the wetting states in the main text, the base radius of the droplet is determined via minimization of the total energy 


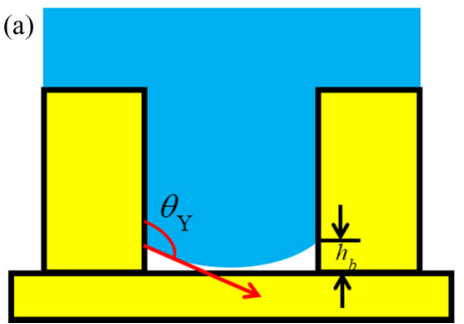

(b)

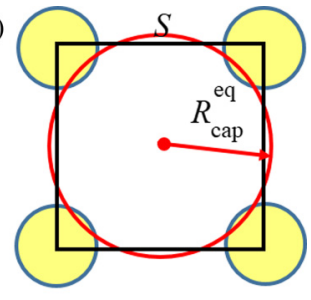

FIG. 7. (a) Illustration of the side view of a droplet in the intermediate composite state with a sagging height and the contact angle between the liquid-vapor interface and the micropillar side walls; (b) the equivalent circle of the projection of the 3D liquid-air interface in a unit cell.

under the constraint of fixed volume, which, in CB state, can be written as

$$
V_{\mathrm{CB}}=\frac{\pi}{3} R_{\mathrm{CB}}^{3} \mu\left(\theta_{\mathrm{CB}}\right)=V_{0},
$$

where $\mu(\theta)=(2+\cos \theta)(1-\cos \theta)^{2} / \sin ^{3} \theta$ is a dimensionless function, and $R_{\mathrm{CB}}$ and $\theta_{\mathrm{CB}}$ are the base radius and the apparent contact angle of the droplet, respectively. Such an equation can also be rewritten as

$$
R_{\mathrm{CB}}=\left[\frac{3 V_{0}}{\pi \mu\left(\theta_{\mathrm{CB}}\right)}\right]^{1 / 3} .
$$

When a droplet on a microstructured surface reaches the intermediate state, its volume can be divided into two parts, i.e., the one on the top of the patterned surface and the other penetrating into the interspacing of the pillars. The volume of the spherical cap above the patterned surface is given by

$$
V_{\text {top }}^{\text {inter }}=\frac{\pi}{3} R_{\text {inter }}^{3} \mu\left(\theta_{\text {inter }}\right) .
$$

In order to calculate the volume of the droplet that penetrates into the interspacing of the pillars, it is necessary to determine the profile of the liquid-air interface. When a droplet is in the intermediate composite state, the liquid-air interface invading a unit cell of micropillar-patterned surface is a curved surface, also referring to sagging liquid. In the absence of external electric field, it has been reported that the contact angle between the curved liquid-air interface and the vertical side wall of the pillars [see Fig. 7(a)] is equal to the intrinsic contact angle $\theta_{\mathrm{Y}}$, when the side walls of pillars are vertical [63]. Even though the side wall of the pillars are curved, at the equilibrium state, the above conclusion still holds [64]. In the presence of an external electric field, the height of the curved liquid-air interface, which is hung over the micropillar side walls, varies with the change of the applied voltage. It has also been experimentally reported that the application of an external field does not change the contact angle considerably [29]. Furthermore, due to the fact that the droplet volume filling the space around the pillars is much smaller than that of the spherical cap above the patterned surface, the variation of the liquid-air interface area underneath the top spherical droplet cap can be neglected in comparison with the liquid-air interface area on the top of the patterned surface. For simplicity, we assume that the contact angle between the curved liquid-air interface and the vertical side wall of the pillars is equal to the Young's contact angle $\theta_{\mathrm{Y}}$ and remains unchanged, as treated in Ref. [31].

It is also reasonable to treat the shape of the liquid-air interface in a unit cell between the pillars as a spherical cap because of the fact that the droplet volume filling the space around the pillars is much smaller than that of the spherical cap above the patterned surface, as shown in Fig. 7(a). Here it is worth noting that the projection of the 3D liquid-air interface in a unit cell is not a circular one. For simplicity, an equivalent base radius $R_{\text {cap }}^{\mathrm{eq}}$ is defined for the projection of the liquid-air interface in a unit cell such that the base area of the actual interface is equal to a circular area of radius $R_{\text {cap }}^{\text {eq }}$ [see Fig. 7(b)], which can be calculated as [31],

$$
\pi\left(R_{\text {cap }}^{\text {eq }}\right)^{2}=S^{2}\left(1-\phi_{s}\right) \Rightarrow R_{\text {cap }}^{\text {eq }}=S\left(\frac{1-\phi_{s}}{\pi}\right)^{1 / 2} .
$$

Thus, the height of the corresponding spherical cap is calculated as

$$
h_{\text {cap }}^{\text {eq }}=R_{\text {cap }}^{\text {eq }} \frac{1-\cos \left(\theta_{\mathrm{Y}}-\pi / 2\right)}{\sin \left(\theta_{\mathrm{Y}}-\pi / 2\right)} .
$$

The penetration height $h$ in the main text can be obtained as $h=H-h_{\text {cap }}^{\text {eq }}$. Then the droplet volume underneath the top spherical cap corresponding to the volume penetrated into the interspacing of the pillars is given by

$$
V_{\text {bottom }}^{\text {inter }}=\frac{\pi}{3} R_{\text {inter }}^{2}\left(1-\phi_{s}\right)\left[3 h+R_{\text {cap }}^{\text {eq }} \mu\left(\theta_{\mathrm{Y}}-\frac{\pi}{2}\right)\right] \text {. }
$$

Given this, the base radius $R_{\text {mid }}$ of a droplet in the intermediate state can be found by solving the following equation,

$$
\begin{aligned}
V_{0}= & \frac{\pi}{3} R_{\text {inter }}^{3} \mu\left(\theta_{\text {inter }}\right) \\
& +\frac{\pi}{3} R_{\text {inter }}^{2}\left(1-\phi_{s}\right)\left[3 h+R_{\text {cap }}^{\mathrm{eq}} \mu\left(\theta_{\mathrm{Y}}-\frac{\pi}{2}\right)\right] .
\end{aligned}
$$

Similarly, when a droplet on a pore-patterned surface gets into intermediate state, the calculation of its base radius can be done in the same manner as above so long as we replace the effective capillary radius of the spherical cap of the bottom part by the radius of the pore $R$.
[1] D. Quéré, Non-sticking drops, Rep. Prog. Phys. 68, 2495 (2005).

[2] D. Quéré, Wetting and roughness, Annu. Rev. Mater. Res. 38, 71 (2008).

[3] C. Choi and C. J. Kim, Large Slip of Aqueous Liquid Flow Over a Nanoengineered Superhydrophobic Surface, Phys. Rev. Lett. 96, 066001 (2006).
[4] P. Joseph, C. Cottin-Bizonne, J.-M. Benoît, C. Ybert, C. Journet, P. Tabeling, and L. Bocquet, Slippage of Water Past Superhydrophobic Carbon Nanotube Forests in Microchannels, Phys. Rev. Lett. 97, 156104 (2006).

[5] A. Steinberger, C. Cottin-Bizonne, P. Kleimann, and E. Charlaix, High friction on a bubble mattress, Nat. Mater. 6, 665 (2007). 
[6] R. Blossey, Self-cleaning surfaces virtual realities, Nat. Mater. 2, 301 (2003).

[7] K. M. Wisdom, J. A. Watson, X. Qu, F. Liu, G. S. Watson, and C. H. Chen, Selfcleaning of superhydrophobic surfaces by selfpropelled jumping condensate, Proc. Natl. Acad. Sci. USA 110, 7992 (2013).

[8] A. Lafuma and D. Quere, Superhydrophobic states, Nat. Mater. 2, 457 (2003).

[9] M. Nosonovsky and B. Bhushan, Biomimetic superhydrophobic surfaces: Multiscale approach, Nano Lett. 7, 2633 (2007).

[10] R. Truesdell, A. Mammoli, P. Vorobieff, F. van Swol, and C. J. Brinker, Drag Reduction on A Patterned Superhydrophobic Surface, Phys. Rev. Lett. 97, 044504 (2006).

[11] J. B. Boreyko and C. H. Chen, Self-Propelled Dropwise Condensate on Superhydrophobic Surfaces, Phys. Rev. Lett. 103, 184501 (2009).

[12] R. H. Siddique, G. Gomard, and H. Holscher, The role of random nanostructures for the omnidirectional anti-reflection properties of the glasswing butterfly, Nat. Commun. 6, 301 (2015).

[13] E. P. Ivanova, J. Hasan, H. K. Webb, V. K. Truong, G. S. Watson, J. A. Watson, V. A. Baulin, S. Pogodin, J. Y. Wang, M. J. Tobin, C. Lobbe, and R. J. Crawford, Natural bactericidal surfaces: mechanical rupture of pseudomonas aeruginosa cells by cicada wings, Small 8, 2489 (2012).

[14] E. P. Ivanova, J. Hasan, H. K. Webb, G. Gervinskas, S. Juodkazis, V. K. Truong, A. H. F. Wu, R. N. Lamb, V. A. Baulin, G. S. Watson, J. A. Watson, D. E. Mainwaring, and R. J. Crawford, Bactericidal activity of black silicon, Nat. Commun. 4, 359 (2013).

[15] X. L. Li, Bactericidal mechanism of nanopatterned surfaces, Phys. Chem. Chem. Phys. 18, 1311 (2016).

[16] K. Xiao, X. Z. Cao, X. Chen, H. Z. Hu, and C. X. Wu, Bactericidal efficacy of nanopatterned surface tuned by topography, J. Appl. Phys. 128, 064701 (2020).

[17] R. N. Wenzel, Resistance of solid surfaces to wetting by water, Ind. Eng. Chem. 28, 988 (1936).

[18] A. B. D. Cassie and S. Baxter, Wettability of porous surface, Trans. Faraday Soc. 40, 546 (1944).

[19] Z. Yoshimitsu, A. Nakajima, T. Watanabe, and K. Hashimoto, Effects of surface structure on the hydrophobicity and sliding behavior of water droplets, Langmuir 18, 5818 (2002).

[20] B. Majhy, V. P. Singh, and A. K. Sen, Understanding wetting dynamics and stability of aqueous droplet over superhydrophilic spot surrounded by superhydrophobic surface, J. Colloid Interface Sci. 565, 582 (2020).

[21] P. Tsai, R. G. H. Lammertink, M. Wessling, and D. Lohse, Evaporation-Triggered Wetting Transition for Water Droplets Upon Hydrophobic Microstructures, Phys. Rev. Lett. 104, 116102 (2010).

[22] X. M. Chen, R. Y. Ma, J. T. Li, C. L. Hao, W. Guo, B. L. Luk, S. C. Li, S. H. Yao, and Z. K. Wang, Evaporation of Droplets on Superhydrophobic Surfaces: Surface Roughness and Small Droplet Size Effects, Phys. Rev. Lett. 109, 116101 (2012).

[23] H. C. M. Fernandes, M. H. Vainstein, and C. Brito, Modeling of droplet evaporation on superhydrophobic surfaces, Langmuir 31, 7652 (2015).

[24] A. Giacomello, M. Chinappi, S. Meloni, and C. M. Casciola, Metastable Wetting on Superhydrophobic Surfaces: Continuum and Atomistic Views of the Cassie-Baxter-Wenzel Transition, Phys. Rev. Lett. 109, 226102 (2012).

[25] P. Lv, Y. Xue, Y. Shi, H. Lin, and H. Duan, Metastable States and Wetting Transition of Submerged Superhydrophobic Structures, Phys. Rev. Lett. 112, 196101 (2014).

[26] A. Sudeepthi, L. Yeo, and A. K. Sen, Cassie-Wenzel wetting transition on nanostructured superhydrophobic surfaces induced by surface acoustic waves, Appl. Phys. Lett. 116, 093704 (2020).

[27] E. Bormashenko, R. Pogreb, G. Whyman, Y. Bormashenko, and M. Erlich, Vibration-induced Cassie-Wenzel wetting transition on rough surfaces, Appl. Phys. Lett. 90, 201917 (2007).

[28] J. B. Boreyko and C. H. Chen, Restoring Superhydrophobicity of Lotus Leaves with Vibration-Induced Dewetting, Phys. Rev. Lett. 103, 174502 (2009).

[29] G. Manukyan, J. M. Oh, D. van den Ende, R. G. H. Lammertink, and F. Mugele, Electrical Switching of Wetting States on Superhydrophobic Surfaces: A Route Towards Reversible Cassie-to-Wenzel Transitions, Phys. Rev. Lett. 106, 014501 (2011).

[30] J. M. Oh, G. Manukyan, D. van den Ende, and F. Mugele, Electric-field-driven instabilities on superhydrophobic surfaces, Europhys. Lett. 93, 56001 (2011).

[31] R. Roy, J. A. Weibel, and S. V. Garimella, Re-entrant cavities enhance resilience to the Cassie-to-Wenzel state transition on superhydrophobic surfaces during electrowetting, Langmuir 34, 12787 (2018).

[32] B. X. Zhang, S. L. Wang, and X. D. Wang, Wetting transition from the Cassie-Baxter state to the Wenzel state on regularly nanostructured surfaces induced by an electric field, Langmuir 35, 662 (2019).

[33] F. Mugele and J. C. Baret, Electrowetting: From basics to applications, J. Phys.: Condens Matter 17, R705 (2005).

[34] W. C. Nelson and C. J. Kim, Droplet actuation by electrowetting-on-dielectric (EWOD): A review, J. Adhes. Sci. Technol. 26, 1747 (2012).

[35] L. Q. Chen and E. Bonaccurso, Electrowetting - from statics to dynamics, Adv. Colloid Interface Sci. 210, 2 (2014).

[36] M. G. Pollack, A. D. Shenderov, and R. B. Fair, Electrowettingbased actuation of droplets for integrated microfluidics, Lab Chip 2, 96 (2002).

[37] V. Bahadur and S. V. Garimella, Electrowetting-based control of static droplet states on rough surfaces, Langmuir 23, 4918 (2007).

[38] A. Cavalli, D. J. Preston, E. Tio, D. W. Martin, N. Miljkovic, E. N. Wang, F. Blanchette, and J. W. M. Bush, Electrically induced drop detachment and ejection, Phys. Fluids 28, 022101 (2016).

[39] Q. Vo and T. Tran, Critical Conditions for Jumping Droplets, Phys. Rev. Lett. 123, 024502 (2019).

[40] Q. G. Wang, M. Xu, C. Wang, J. P. Gu, N. Hu, J. F. Lyu, and W. Yao, Actuation of a nonconductive droplet in an aqueous fluid by reversed electrowetting effect, Langmuir 36, 8152 (2020).

[41] K. Xiao and C. X. Wu, Curvature effect of electrowettinginduced droplet detachment, J. Appl. Phys. 129, 234701 (2021).

[42] S. Berry, T. Fedynyshyn, L. Parameswaran, and A. Cabral, Switchable electrowetting of droplets on dual-scale structured surfaces, J. Vac. Sci. Technol. B 30, 06F801 (2012). 
[43] Y. Chen, Y. Suzuki, and K. Morimoto, Electrowettingdominated instability of Cassie droplets on superlyophobic pillared surfaces, Langmuir 35, 2013 (2019).

[44] A. M. Miqdad, S. Datta, A. K. Das, and P. K. Das, Effect of electrostatic incitation on the wetting mode of a nano-drop over a pillar-arrayed surface, RSC Adv. 6, 110127 (2016).

[45] M. Amabili, A. Giacomello, S. Meloni, and C. M. Casciola, Unraveling the Salvinia paradox: Design principles for submerged superhydrophobicity, Adv. Mater. Interfaces 2, 1500248 (2015).

[46] P. Lv, Y. Xue, H. Liu, Y. Shi, P. Xi, H. Lin, and H. Duan, Symmetric and asymmetric meniscus collapse in wetting transition on submerged structured surfaces, Langmuir 31, 1248 (2015).

[47] Y. He, Q. Zhou, S. Wang, R. Yang, C. Jiang, and W. Yuan, In situ observation of dynamic wetting transition in re-entrant microstructures, Langmuir 33, 3949 (2017).

[48] N. A. Patankar, Consolidation of hydrophobic transition criteria by using an approximate energy minimization approach, Langmuir 26, 8941 (2010).

[49] W. Ren, Wetting transition on patterned surfaces: Transition states and energy barriers, Langmuir 30, 2879 (2014).

[50] M. Amabili, A. Giacomello, S. Meloni, and C. M. Casciola, Collapse of superhydrophobicity on nanopillared surfaces, Phys. Rev. Fluids 2, 034202 (2017).

[51] T. Koishi, K. Yasuoka, S. Fujikawa, T. Ebisuzaki, and X. C. Zeng, Coexistence and transition between Cassie and Wenzel state on pillared hydrophobic surface, Proc. Natl. Acad. Sci. USA 106, 8435 (2009).

[52] W. Gong, Y. Zu, S. Chen, and Y. Yan, Wetting transition energy curves for a droplet on a square-post patterned surface, Sci. Bull. 62, 136 (2017)

[53] G. Pashos, G. Kokkoris, and A. G. Boudouvis, Minimum energy paths of wetting transitions on grooved surfaces, Langmuir 31, 3059 (2015).
[54] C. Rohrs, A. Azimi, and P. He, Wetting on micropatterned surfaces: Partial penetration in the Cassie state and Wenzel deviation theoretically explained, Langmuir 35, 15421 (2019).

[55] T. Liu, Y. Li, X. Li, and W. Sun, Mechanism study on transition of Cassie droplets to Wenzel state after meniscus touching substrate of pillars, J. Phys. Chem. C 121, 9802 (2017).

[56] Y. Xue, S. Chu, P. Lv, and H. Duan, Importance of hierarchical structures in wetting stability on submersed superhydrophobic surfaces, Langmuir 28, 9440 (2012).

[57] Y. Xue, P. Lv, H. Lin, and H. Duan, Underwater superhydrophobicity: Stability, design and regulation, and applications, Appl. Mech. Rev. 68, 030803 (2016).

[58] Y. Li, D. Quéré, C. Lv, and Q. Zheng, Monostable superrepellent materials, Proc. Natl. Acad. Sci. USA 114, 3387 (2017).

[59] K. Xiao, Y. P. Zhao, G. Ouyang, and X. L. Li, An analytical model of nanopatterned superhydrophobic surfaces, J. Coat. Technol. Res. 14, 1297 (2017).

[60] V. Bahadur and S. V. Garimella, Electrowetting-based control of droplet transition and morphology on artificially microstructured surfaces, Langmuir 24, 8338 (2008).

[61] Y.-P. Zhao and Q. Yuan, Statics and dynamics of electrowetting on pillar-arrayed surfaces at the nanoscale, Nanoscale 7, 2561 (2015).

[62] B. Rofman, S. Dehe, V. Frumkin, S. Hardt, and M. Bercovici, Intermediate states of wetting on hierarchical superhydrophobic surfaces, Langmuir 36, 5517 (2020).

[63] B. Hou, C. Wu, X. Li, J. Huang, and M. Chen, Contact line-based model for the Cassie-Wenzel transition of a sessile droplet on the hydrophobic micropillar-structured surfaces, Appl. Surf. Sci. 542, 148611 (2021).

[64] H. Y. Guo, B. Li, and X. Q. Feng, Stability of Cassie-Baxter wetting states on microstructured surfaces, Phys. Rev. E 94, 042801 (2016). 